\title{
DIFICULDADES NA APRENDIZAGEM ESCOLAR, ATRASO MOTOR E PRÁTICA DE ATIVIDADE FÍSICA: REVISÃO SISTEMÁTICA
}

\author{
Alexandre Nascimento ${ }^{1}$ \\ Gabriel Sena Nascimento ${ }^{2}$
}

NASCIMENTO, A.; NASCIMENTO, G. S. Dificuldades na aprendizagem escolar, atraso motor e prática de atividade física: Revisão sistemática. Arq. Cienc. Saúde UNIPAR, Umuarama, v. 24, n. 1, p. 61-66, jan./abr. 2020.

\begin{abstract}
RESUMO: O processo humano do aprendizado é marcado pelo dinamismo e complexidade, estruturado a partir do ato motor e da percepção, dando origem à cognição. Contudo, a influência da prática de atividade física e das aulas de educação física no desenvolvimento da função cognitiva ainda não são consenso entre os pesquisadores. Sendo assim, este estudo tem como objetivo verificar se crianças com problemas na aprendizagem escolar apresentam maior atraso motor e se a prática de atividade física contribui para melhora do processo cognitivo de aprendizagem. Foram selecionados neste estudo 8 artigos que investigaram $(n=835)$ escolares de 6 a 12 anos. Os resultados demonstraram que a dificuldade na aprendizagem escolar está relacionada ao maior atraso motor e que grupos que participaram de atividades físicas e das aulas de educação física melhoraram o desempenho das funções cognitivas de aprendizagem. Portanto, torna-se fundamental a prática de atividade física e a participação das aulas de educação física, devido sua contribuição no processo de fortalecimento do desenvolvimento infantil.
\end{abstract}

PALAVRAS-CHAVE: Aprendizagem escolar. Crianças. Desenvolvimento motor.

\section{DIFFICULTIES IN SCHOOL LEARNING, MOTOR DELAY AND THE PRACTICE OF PHYSICAL ACTIVITY: A SYSTEMATIC REVIEW}

\begin{abstract}
Human learning is marked by dynamism and complexity. It is structured from the human motor skills and perception, and thus originating cognition. However, there is no agreement among researchers on the influence of physical activity and physical education classes on the development of cognitive function. Therefore, this work aims at verifying if schoolchildren with learning disabilities present greater human motor delay and if physical activity contributes towards a better cognitive leaning process. Moreover, this study also aims at verifying if physical activity improves cognitive learning. In this study, a total of eight (8) journal articles were chosen, which investigated $(\mathrm{n}=835)$ students aged from 6 to 12 years old. The results showed that learning disability is related to a higher human motor delay. It also demonstrated that groups which participated in physical activities and in physical education classes presented better human motor skills and better performance of the cognitive functions in learning. Thus, it can be concluded that physical activity and physical education classes play an important role on the progress of children development.
\end{abstract}

KEYWORDS: School learning. Children. Human motor development.

Introdução

O desenvolvimento humano é influenciado por vários aspectos, com isto encontramos diversos fatores que são importantes nesse processo, tais como: aspectos cognitivos, afetivos, motores e psicossociais, dos quais se desenvolvem em um processo contínuo durante toda a vida (NETO, 2002).

O processo humano de aprendizado é outro fator de destaque, sendo marcado pelo dinamismo e complexidade, o qual é estruturado a partir do ato motor e da percepção, dando origem a cognição (GUARDIOLA; FERREIRA; ROTTAOTTA, 1998).

A melhora nas funções motoras que a criança recebe está ligada diretamente à maturação do córtex, estando o seu desenvolvimento intimamente ligado aos estímulos ambientais que recebe. No entanto, mudanças nas áreas específicas do sistema nervoso central, relacionadas com a noção do esquema corporal, do espaço-tempo e da lateralidade, constituem as bases neuropatológicas das desordens perceptivo motoras, das quais podem resultar em problemas na aprendizagem da leitura, escrita e cálculo (ROCHA; TUDELLA, 2002)

Uma das fases mais significativas na vida do ser humano é justamente a fase do desenvolvimento infantil (ARAUJO; MINERVINO, 2008). Na segunda infância da idade escolar há um avanço nas aptidões físicas e motora, é quando a criança desenvolve consciência de si e do mundo exterior, conquistando sua independência e adaptação social, caracterizando um avanço na aprendizagem (LINEBURGUER et al., 2004). Com isso percebemos que a aquisição de um bom controle motor permite à criança construir as noções básicas para seu desenvolvimento intelectual.

Dando-se oportunidades para crianças desempenhar suas habilidades, estas por sua vez serão desenvolvidas, por isso, a Educação Física adquire papel importante na medida em que pode estruturar o ambiente adequado para a criança, oferecendo experiências e resultando como grande promotora do desenvolvimento humano, em especial ao desenvolvimento motor (SCHIRMER; FONTOURA; NUNES, 2004).

Com isso, é de suma importância à interação do corpo de forma completa no processo do desenvolvimento escolar para se entender alguns problemas relacionados à escrita e à linguagem, principalmente os relacionados a ação motora, nos desafiando a conhecer um pouco o que uma "pedagogia" do movimento humano teria a nos ensinar (NETO et al., 2005; PELLEGRINI et al., 2003).

Trabalhando em conjunto com a maturação biológica, o desenvolvimento das capacidades motoras fortalece no ser humano condições de movimentar-se de maneira contínua, em ritmos variados e de diversas formas, porém, temos que considerar que as mudanças ocorridas no sistema motor da criança ocorrem simultaneamente ao desenvolvimento do 
sistema cognitivo. Sendo assim, não há como separar esses dois aspectos do desenvolvimento, pois ambos agem em conjunto no mesmo organismo (CARDEAL et al., 2013).

Nesta tentativa de observar a relação entre o sistema cognitivo e a prática de atividade física, alguns pesquisadores observaram benefícios adquiridos pela cognição advinda das atividades físicas em crianças com idade escolar (CARDEAL et al., 2013; HILLMAN; CASTELLI; BUCK, 2005).

Com isso, a avaliação motora torna-se um instrumento que ajuda no reconhecimento de dados relacionados ao desenvolvimento motor da criança e sugere estratégias conjuntas de atividades relacionadas às necessidades específicas de cada uma delas (MEDINA-PAPST; MARQUES, 2010).

Assim, grande parte dos problemas de aprendizagem encontram-se sobre as áreas de percepção, atenção, memória, associação e fixação de informações, os quais podem permanecer na vida jovem e adulta (NETO et al., 2007). Crianças com dificuldades de aprendizagem em leitura e escrita, na sua maioria, possuem a mesma forma de relacionar-se com as outras áreas trabalhadas no contexto escolar (BALTAZAR, 2001).

Destarte, o objetivo deste estudo é verificar se crianças com problemas na aprendizagem escolar apresentam maior atraso motor e se a prática de atividade física contribui para melhora do processo cognitivo de aprendizagem.

\section{Método}

Trata-se de uma revisão sistemática da literatura nacional e internacional sobre as associações entre dificuldade na aprendizagem e atraso motor em escolares. Para a realização do estudo seguiu-se a Declaração PRISMA - Prefere Reporting Itens for Sistematic Reviews and Meta-Analyses, um protocolo que orienta a construção de revisões sistemáticas e consiste em uma lista de verificação de 27 itens e um diagrama de fluxo de quatro fases (URRUTIA; TORTA; BONFILL, 2005). Para selecionar os descritores que serão usados na busca utilizou-se, o MeSH (Medical Subject Headings), que são ferramentas de consulta da terminologia usada e reconhecida mundialmente, organizando e padronizando a busca de determinados temas. Após esse levantamento, as palavras-chaves e os operadores booleanos utilizados na busca da revisão foram de acordo com a estratégia do (PECOS) Population, Exposure, Comparator, Outcome, Study design.

Quadro 1. Critérios de inclusão e exclusão dos estudos

\begin{tabular}{ccc}
\hline & $\begin{array}{c}\text { Critérios de } \\
\text { inclusão }\end{array}$ & $\begin{array}{c}\text { Critérios de } \\
\text { exclusão }\end{array}$ \\
\hline Participate & Escolares & Adultos \\
& Dificuldade de & \\
Exposure & aprendizagem e / ou \\
& Atraso Motor & \\
& Com intervenção & \\
Comparison & ou nenhuma & - \\
& intervenção &
\end{tabular}

\begin{tabular}{|c|c|}
\hline Termos & Descritores \\
\hline $\begin{array}{l}\text { Dificuldade na } \\
\text { Aprendizagem }\end{array}$ & $\begin{array}{c}\text { "Learning Disorders" OR "Learning } \\
\text { Disabilities" OR Difficulties, } \\
\text { Handwriting OR "Scholastic Skills" OR } \\
\text { "Development Disorders" }\end{array}$ \\
\hline Atraso motor & $\begin{array}{l}\text { "Psychomotor Performance" OR "Visual } \\
\text { Perception" OR Motor Skills OR "Visual } \\
\text { Motor Coordination" OR "Psychomotor } \\
\text { Performance" OR "Sensory Motor } \\
\text { Learning" OR "Psychomotor Skills" } \\
\text { OR "Perceptual Motor Learning” OR } \\
\text { Coordination, "Perceptual Motor" OR } \\
\text { "Motor Development” OR "Perceptual } \\
\text { Motor Learning" OR "Perceptual } \\
\text { Development" OR "Perceptual Motor } \\
\text { Coordination" }\end{array}$ \\
\hline Escolares & $\begin{array}{l}\text { Child OR children OR students OR } \\
\text { "Young Children" OR "Elementary } \\
\text { School Children" }\end{array}$ \\
\hline Combinações & $\# 1$ AND \#2 AND \#3 \\
\hline
\end{tabular}

Foram incluídos apenas artigos científicos publicados sobre dificuldade na aprendizagem e atraso motor de escolares, publicados em inglês, português e espanhol, sendo excluídos artigos de revisão, dissertações, teses ou resumos publicados em anais de congressos. Os procedimentos de busca e seleção dos artigos foram realizados por dois pesquisadores de forma independente e em caso de discordância, um terceiro foi solicitado para parecer final.

$\mathrm{Na}$ etapa inicial foi realizada a exclusão dos artigos duplicados e com base na leitura dos títulos. Em seguida, foram lidos os resumos daqueles não excluídos na primeira 
etapa. Na fase seguinte, todos os artigos selecionados pelo resumo foram obtidos na íntegra e posteriormente examinados de acordo com os critérios de inclusão estabelecidos. Foi utilizado critério temporal de (2007 a 2019) para inclusão dos estudos.

\section{Resultados}

A busca manual e nas bases de dados identificaram 810 artigos. Foram excluídos 71 duplicados, 591 pelo título, 148 artigos após leitura dos resumos e 55 após leitura na íntegra. Ao final, foram incluídos 8 estudos. O processo de seleção está apresentado no fluxograma da Figura 1.

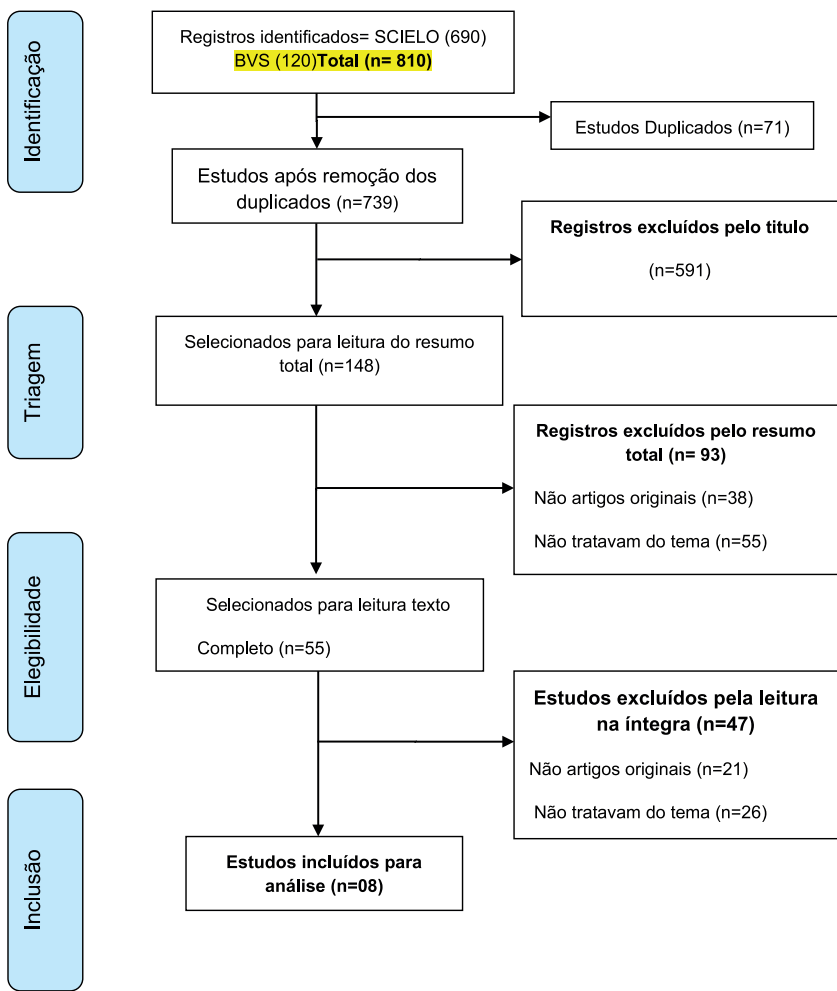

Figura 1: Fluxograma de busca e seleção dos estudos na presente revisão.

A primeira publicação registrada nos periódicos investigados ocorreu no ano de 2007 e a última em 2015, a partir desta data pouco material foi encontrado. Entre os artigos relacionados à temática, houve $(n=8)$ com abordagem quantitativa. As publicações estão nas revistas: Revista Brasileira Ciência e Movimento (2007), Research in Developmental Disabilities (2011), Revista Semestral da Associação Brasileira de Psicologia Escolar e Educacional (2011), Temas sobre Desenvolvimento (2012) e Revista Motricidade (2012), CEFAC (2013), The Physical Educator (2014) e Revista Psicopedagogia (2015). Em relação ao número de participantes nas pesquisas quantitativas foram computados 865 sujeitos. Em relação ao sexo, todos os estudos investigaram o sexo masculino e feminino (Quadro 3).
Quadro 3: Resultado metodológico: Ano, tipo de estudo, revista, tamanho da amostra e sexo.

\begin{tabular}{|c|c|c|c|c|}
\hline Referência & Revista & $\begin{array}{l}\text { Tipo de } \\
\text { estudo }\end{array}$ & Total & Sexo \\
\hline $\begin{array}{l}\text { Neto et al. } \\
\quad(2007)\end{array}$ & $\begin{array}{c}\text { Revista Brasileira } \\
\text { Ciência e } \\
\text { Movimento }\end{array}$ & Quantitativo & 289 & $\mathrm{M} / \mathrm{F}$ \\
\hline $\begin{array}{l}\text { Cheng et } \\
\text { al. } \\
(2011)\end{array}$ & $\begin{array}{l}\text { Research in } \\
\text { Developmental } \\
\text { Disabilities }\end{array}$ & Quantitativo & 130 & $\mathrm{M} / \mathrm{F}$ \\
\hline $\begin{array}{l}\text { Neto et al. } \\
\quad(2011)\end{array}$ & $\begin{array}{l}\text { Revista semestral } \\
\text { da Associação } \\
\text { Brasileira de } \\
\text { Psicologia Escolar } \\
\text { e Educacional }\end{array}$ & Quantitativo & 39 & $\mathrm{M} / \mathrm{F}$ \\
\hline $\begin{array}{l}\text { Neto et al. } \\
\text { (2013) }\end{array}$ & CEFAC & Quantitativo & 166 & $\mathrm{M} / \mathrm{F}$ \\
\hline $\begin{array}{c}\text { Cardeal et } \\
\text { al. } \\
(2013)\end{array}$ & $\begin{array}{c}\text { Revista } \\
\text { Motricidade }\end{array}$ & Quantitativo & 80 & $\mathrm{M} / \mathrm{F}$ \\
\hline $\begin{array}{l}\text { Neto et al. } \\
\text { (2013) }\end{array}$ & $\begin{array}{c}\text { Temas sobre } \\
\text { Desenvolvimento }\end{array}$ & Quantitativo & 01 & $\mathrm{M} / \mathrm{F}$ \\
\hline $\begin{array}{l}\text { Li et al. } \\
\text { (2014) }\end{array}$ & $\begin{array}{c}\text { The Physical } \\
\text { Educator }\end{array}$ & Quantitativo & 83 & $\mathrm{M} / \mathrm{F}$ \\
\hline $\begin{array}{l}\text { Carvalho; } \\
\text { Ciasca; } \\
\text { Rodrigues } \\
\quad(2015)\end{array}$ & $\begin{array}{c}\text { Revista } \\
\text { Psicopedagogia }\end{array}$ & Quantitativo & 25 & $\mathrm{M} / \mathrm{F}$ \\
\hline
\end{tabular}

Legenda. M:Masculino,F:Feminino. Fonte: elaborado pelos autores, 2019.

Neto et al. (2007) mostram que alunos com dificuldades na aprendizagem escolar apresentam atraso no desenvolvimento motor, bem como condições biopsicossociais adversas, demonstrando uma possível relação entre estes fatores.

Cheng et al. (2011) apresentam associação entre dificuldade motora e baixo desempenho na escrita.

Neto et al. (2011) os dados sugerem que o desenvolvimento das capacidades motoras, em especial do esquema corporal, são fundamentais para o desenvolvimento de habilidades essenciais à aprendizagem escolar.

Neto et al. (2013) mostraram a importância de trabalhar com o corpo na escola por meio da educação física e da contribuição de projetos interdisciplinares.

Cardeal et al. (2013) apresentam a importância da educação física no ensino fundamental como meio efetivo de auxílio para as aprendizagens motoras e cognitivas.

Neto et al. (2013) verificaram que de todas as variáveis do desempenho da leitura e da escrita, foram maiores para o grupo das crianças com dominância lateral completa do que para o grupo das crianças com lateralidade cruzada.

Li et al. (2014)verificaram melhorias na habilidade de escrita no grupo participante das atividades físicas específicas.

Carvalho; Ciasca; Rodrigues (2015) constatou que o grupo com Transtorno de déficit de atenção com Hiperatividade (TDAH) teve pior desempenho, porém a diferença estatística significativa foi encontrada apenas em esquema corporal quando se comparou o grupo TDAH com o grupo com transtorno de aprendizagem. 
Quadro 4: Descrição do título, objetivo e principais resultados

\begin{tabular}{|c|c|c|c|}
\hline Referência & Título & Objetivos & Principais resultados \\
\hline $\begin{array}{l}\text { Neto et al. } \\
\quad(2007)\end{array}$ & $\begin{array}{l}\text { DM de Crianças com Indi- } \\
\text { cadores de Dificuldades na } \\
\text { Aprendizagem Escolar. }\end{array}$ & $\begin{array}{l}\text { Avaliar o desenvolvimento motor } \\
\text { e as características psicossociais } \\
\text { de crianças com indicadores de } \\
\text { aprendizagem escolar. }\end{array}$ & $\begin{array}{l}\text { Crianças com dificuldade escolar apresen- } \\
\text { tam importante atraso no DM. }\end{array}$ \\
\hline $\begin{array}{l}\text { Cheng et al. } \\
\text { (2011) }\end{array}$ & $\begin{array}{l}\text { Reading and writing per- } \\
\text { formances of children 7-8 } \\
\text { years of age with develop- } \\
\text { mental coordination disor- } \\
\text { der in Taiwan. }\end{array}$ & $\begin{array}{l}\text { Analisar o desempenho de leitura } \\
\text { e escrita de crianças com Trans- } \\
\text { torno do Desenvolvimento da } \\
\text { Coordenação. }\end{array}$ & $\begin{array}{l}\text { As crianças com dificuldade motora tive- } \\
\text { ram escores significativamente mais baixos } \\
\text { na escrita do que as crianças com desenvol- } \\
\text { vimento típico. O desempenho de escrita é } \\
\text { mais baixo nas crianças com dificuldade } \\
\text { motora do que nas crianças com desenvol- } \\
\text { vimento típico. Não foi encontrado nenhu- } \\
\text { ma diferença no desempenho de leitura. }\end{array}$ \\
\hline $\begin{array}{l}\text { Neto et al. } \\
\quad(2011)\end{array}$ & $\begin{array}{l}\text { O esquema corporal de } \\
\text { crianças com dificuldade } \\
\text { de aprendizagem. }\end{array}$ & $\begin{array}{l}\text { Verificar o desenvolvimento do } \\
\text { esquema corporal de escolares } \\
\text { na faixa etária de } 6 \text { a } 10 \text { anos } \\
\text { com queixa de dificuldades de } \\
\text { aprendizagem, encaminhados ao } \\
\text { Núcleo de Avaliação e Interven- } \\
\text { ção Motora - NAIM/LADEHU/ } \\
\text { UDESC. }\end{array}$ & $\begin{array}{l}\text { O déficit aumentou conforme a idade cro- } \\
\text { nológica dos participantes e a classificação } \\
\text { do Quociente Motor Geral e do Quociente } \\
\text { Motor do Esquema Corporal do grupo foi } \\
\text { Muito Inferior. Os dados sugerem que o } \\
\text { desenvolvimento das capacidades motoras, } \\
\text { em especial do esquema corporal, é funda- } \\
\text { mental para o desenvolvimento de habili- } \\
\text { dades essenciais à aprendizagem escolar. }\end{array}$ \\
\hline $\begin{array}{l}\text { Neto et al. } \\
\quad(2013)\end{array}$ & $\begin{array}{l}\text { Efeitos da intervenção mo- } \\
\text { tora em uma criança com } \\
\text { transtorno de aspecto de } \\
\text { autismo. }\end{array}$ & $\begin{array}{l}\text { Avaliar o DM de uma criança de } \\
9 \text { anos que apresenta transtorno } \\
\text { global do desenvolvimento com } \\
\text { espectro autista e verificar os } \\
\text { efeitos de uma intervenção mo- } \\
\text { tora. }\end{array}$ & $\begin{array}{l}\text { A educação física tem uma grande contri- } \\
\text { buição em projetos integrados com outras } \\
\text { disciplinas. }\end{array}$ \\
\hline $\begin{array}{l}\text { Cardeal et } \\
\text { al. (2013) }\end{array}$ & $\begin{array}{l}\text { Efeito de um programa es- } \\
\text { colar de estimulação mo- } \\
\text { tora sobre desempenho da } \\
\text { função executiva e atenção } \\
\text { em crianças. }\end{array}$ & $\begin{array}{l}\text { Verificar o efeito da estimulação } \\
\text { motora, nas respostas da função } \\
\text { cognitiva de crianças na faixa } \\
\text { etária de } 6 \text { a } 10 \text { anos. }\end{array}$ & $\begin{array}{l}\text { A educação física foi importante no ensino } \\
\text { fundamental como meio efetivo de auxílio } \\
\text { para as aprendizagens motoras e cogniti- } \\
\text { vas. }\end{array}$ \\
\hline $\begin{array}{l}\text { Neto et al. } \\
\text { (2013) }\end{array}$ & $\begin{array}{l}\text { Lateralidade e o desempe- } \\
\text { nho da leitura e escrita em } \\
\text { escolares. }\end{array}$ & $\begin{array}{l}\text { Analisar o desempenho da leitura } \\
\text { e da escrita em escolares com la- } \\
\text { teralidade cruzada. }\end{array}$ & $\begin{array}{l}\text { O desempenho da leitura e da escrita, fo- } \\
\text { ram maiores para o grupo das crianças com } \\
\text { dominância lateral completa do que com } \\
\text { lateralidade cruzada. }\end{array}$ \\
\hline $\begin{array}{l}\text { Li et al. } \\
(2014)\end{array}$ & $\begin{array}{l}\text { Effects of a Sport Stacking } \\
\text { Intervention on Second- } \\
\text {-Grade Students. }\end{array}$ & $\begin{array}{l}\text { Examinar os efeitos de uma inter- } \\
\text { venção (esporte de empilhamen- } \\
\text { to) sobre a coordenação olho- } \\
\text {-mão, tempo de reação e habili- } \\
\text { dades de escrita manual }\end{array}$ & $\begin{array}{l}\text { Os resultados não foram conclusivos. No } \\
\text { entanto, houve uma tendência para melho- } \\
\text { rias significativas no grupo experimental } \\
\text { sobre o grupo de controle. Foram observa- } \\
\text { das melhorias na habilidade de escrita e no } \\
\text { comportamento em sala de aula. }\end{array}$ \\
\hline $\begin{array}{l}\text { Carvalho; } \\
\text { Ciasca; } \\
\text { Rodrigues } \\
\text { (2015) }\end{array}$ & $\begin{array}{l}\text { Há relação entre desen- } \\
\text { volvimento psicomotor e } \\
\text { dificuldade de aprendiza- } \\
\text { gem? Estudo comparativo } \\
\text { de crianças com transtorno } \\
\text { de déficit de atenção e hi- } \\
\text { peratividade, dificuldade } \\
\text { escolar e transtorno de } \\
\text { aprendizagem. }\end{array}$ & $\begin{array}{l}\text { Avaliar o desempenho psicomo- } \\
\text { tor de crianças (TA), dificuldade } \\
\text { escolar (DE) e (TDH). }\end{array}$ & $\begin{array}{l}\text { Todas as crianças tiveram idade motora } \\
\text { inferior à idade cronológica. Comparando } \\
\text { as habilidades psicomotoras, constatou-se } \\
\text { que o grupo com TDAH teve pior desem- } \\
\text { penho, porém diferença estatisticamente } \\
\text { significativa foi encontrada apenas em } \\
\text { esquema corporal quando se comparou o } \\
\text { grupo TDAH com o grupo com TA. }\end{array}$ \\
\hline
\end{tabular}

Legenda.Transtorno de aprendizagem (TA), Dificuldade escolar (DE), Transtorno do déficit de atenção com hiperatividade (TDAH), Desenvolvimento Motor (DM). Fonte: elaborado pelos autores, 2019. 


\section{Discussão}

O tema dificuldade na aprendizagem escolar e atrasos motores é pouco referenciado na literatura, contudo encontramos sete estudos que analisaram essas variáveis. De acordo com os resultados, os dados nos evidenciaram que existe associação entre dificuldades na aprendizagem e atraso motor.

No estudo de lateralidade cruzada e desempenho da leitura e escrita, evidencia-se que crianças com lateralidade cruzada apresentam desempenho inferior na leitura e escrita quando comparadas às crianças com dominância lateral completa. Estes dados justificam a relevância do desenvolvimento psicomotor na infância como fator essencial no processo de aprendizagem escolar (NETO et al., 2013). Em concordância a este resultado, verificou-se que crianças com indicadores de dificuldades na aprendizagem, matriculadas na rede municipal de Florianópolis/SC, apresentaram lateralidade indefinida (NETO et al., 2007).

Portanto, crianças com lateralidade ainda não definida podem precisar de atenção especial, pois o estabelecimento e o conhecimento da lateralidade são primordiais no desenvolvimento da orientação e relação espacial, interferindo indiretamente na aprendizagem escolar (NETO et al., 2005; RODRIGUES, 2000).

No estudo de Neto et al. (2011), todos os participantes apresentaram atraso no desenvolvimento da Idade motora geral e na idade motora do esquema corporal. Sendo a constituição do esquema corporal fundamental para o desenvolvimento da criança, a percepção do próprio corpo e a percepção deste no espaço e no tempo se torna essenciais para o desenvolvimento harmonioso dos aspectos motores, físicos e cognitivos.

Também analisando os parâmetros motores, observou-se que grande parte apresentou déficit entre a idade cronológica e a idade motora geral, com média de 16 meses, o que pode fomentar o elo existente entre aspectos cognitivos e motores, embora o quociente geral de desenvolvimento tenha apontado à média de normalidade (NETO et al., 2007).

O estudo de Cheng et al. (2011), analisou o desempenho da leitura e escrita de crianças com Transtorno do Desenvolvimento da Coordenação comparando-as com crianças sem problemas motores. Os autores observaram que o desempenho da escrita se mostrou menor nas crianças com Transtorno do Desenvolvimento da Coordenação do que nas crianças com desenvolvimento típico.

O fraco desempenho da escrita das crianças com Transtorno do Desenvolvimento da Coordenação pode ser atribuído à sua dificuldade motora, pois segundo Medina-Papst; Marques (2010) o maior atraso na área de esquema corporal, sugere-se inclusão de tarefas que auxiliem no desenvolvimento integral do aluno, além de atividades motoras especificas com objetivo de uma evolução no desenvolvimento do aprendizado escolar.

Sendo assim, as práticas corporais, os movimentos, são formas de explorar o corpo e suas possibilidades, proporcionando a percepção corporal a partir dessa prática, contribuindo para a formação integral do indivíduo (ANDRADE FILHO; SCHNEIDER, 2009). Assim a prática da educação física é um fator importante no processo de desenvolvimen- to, pois ajuda na melhora do desempenho pleno da criança (NETO et al., 2013).

No estudo sobre a pedagogia do movimento humano, o corpo como objeto de estudo, nos mostra que a criança deve ser estimulada ao movimento, deve adquirir consciência corporal e destreza em algumas habilidades, para realizar as tarefas diárias (NETO et al., 2005; PELLEGRINI et al., 2003). Mostrando que diante deste pressuposto é inegável que a escola deva lembrar, a todo instante, a dimensão corporal e a importância que este assume no processo de aprendizagem (ANDRADE FILHO; SCHNEIDER, 2009; NETO et al., 2013; SCHIRMER; FONTOURA; NUNES, 2004).

A estimulação ao movimento é um fator muito importante, no estudo de Cardeal et al. (2013), foi observado que o grupo que mais participou das atividades físicas, melhorou o aspecto motor e a atenção seletiva, ou seja, o raciocínio ainda que baseado nas operações concretas tornou-se mais rápido, requisitando menos tempo para solucionar um problema.

O estudo de Li et al. (2014) verificou-se o efeito de 14 semanas de intervenção com exercício específicos sobre a qualidade e velocidade da escrita. Os resultados não foram conclusivos, apenas indicaram que o grupo experimental demonstrou melhora superior na velocidade de escrita do que o grupo controle no pós-teste. Estudos descrevem sobre a necessidade de desenvolver mais estudos de intervenção, devido carência de investigação a respeito dos efeitos das intervenções psicomotoras sobre o funcionamento cognitivo (BROWN, 2010; GRISSMER et al., 2010).

Com relação ao estudo de Carvalho; Ciasca; Rodrigues (2015) foi identificado que a idade motora de todos os sujeitos foi inferior à idade cronológica. Tal dado reforça o que é mencionado por Fonseca (1995), de que a motricidade organiza as sensações e percepções que dão origem às aprendizagens mais complexas.

Por fim, os resultados encontrados reforçam evidências apresentadas em outros estudos, de que a dificuldade na aprendizagem escolar é um fator evidente que pode estar associado ao atraso motor e que a prática de atividade física e participação nas aulas de educação física são fortes aliados neste processo de melhora cognitiva e motora.

\section{Conclusão}

Portanto, este estudo verificou que alunos com problemas de aprendizagem escolar apresentaram maior atraso motor e que os grupos que participaram mais das atividades físicas propostas, tais como, aulas de educação física, melhoraram especificamente no desempenho dos testes de função executiva e no desempenho das funções cognitivas de aprendizagem. Com isso conclui-se como fundamental a prática de atividade física e a participação nas aulas de Educação Física como processo de contribuição e de fortalecimento no desenvolvimento infantil.

Contudo, a quantidade de estudos disponíveis e a qualidade do delineamento adotado impede que generalizações possam ser efetuadas. Dessa forma, sugere-se que mais estudos possam analisar esta relação, a fim de contribuir para o avanço da pesquisa. 


\section{Referências}

ANDRADE FILHO, N. F. D.; SCHNEIDER, O. Educação Física para a Educação Infantil: conhecimento e especificidade. Aracajú: Editora da UFSE, 2009. 229 p.

ARAUjO, M. R.; MINERVINO, C. A. D. S. M. Avaliação cognitiva: leitura, escrita e habilidades relacionadas. Psicologia em Estudo, v. 4, n. 13, p. 859-865, 2008.

BALTAZAR, M. O. Tratamento das dificuldades de aprendizagem de leitura e escrita à luz da psicopedagogia construtivista. Reflexão e Ação, 9, n. 1, p. 37-46, 2001.

BROWN, C. G. Improving fine motor skills in young children: an intervention study. Educational Psychology in Practice, 26, n. 3, p. 269-278, 2010/09/01 2010.

CARDEAL, C. M. et al. Efeito de um programa escolar de estimulação motora sobre desempenho da função executiva e atenção em crianças. Motricidade, v. 9, n. 3, p. 44-56, 2013.

CARVALHO, M. C.; CIASCA, S. M.; RODRIGUES, S. D. D. Há relação entre desenvolvimento psicomotor e dificuldade de aprendizagem?: Estudo comparativo de crianças com transtorno de déficit de atenção e hiperatividade, dificuldade escolar e transtorno de aprendizagem. Revista Psicopedagogia, v. 32, n. 99, p. 293-301, 2015.

CHENG, H.-C. et al. Reading and writing performances of children 7-8 years of age with developmental coordination disorder in Taiwan. Research in Developmental Disabilities, v. 32, n. 6, p. 2589-2594, 2011.

FONSECA, V. Manual de Observação Psicomotora Significação psiconeurológica dos fatores psicomotores. Porto Alegre: Artes Médicas, 1995. 324 p.

GRISSMER, D. et al. Fine motor skills and early comprehension of the world: Two new school readiness indicators. Developmental Psychology, v. 46, n. 5, p. 10081017, 2010.

GUARDIOLA, A.; FERREIRA, L. T. C.; ROTTAOTTA, N. T. Associação entre desempenho das funções corticais e alfabetização em uma amostra de escolares de primeira série de Porto Alegre. Arquivos de Neuro-Psiquiatria, v. 56, n. 2, p. 281-288, 1998.

HILlMAN, C.; CASTELLI, D.; BUCK, S. Aerobic Fitness and Neurocognitive Function in Healthy Preadolescent Children. Medicine and science in sports and exercise, v. 37, n. 11, p. 1967-1974, 2005.

LI, Y. et al. The Effect of a Sport Stacking Intervention on Handwriting With Second Grade Students. The Physical Educator, v. 71, n. 1, p. 59-71, 2014.

LINEBURGUER, A. A. et al. Desenvolvimento motor de crianças asmáticas. Temas sobre Desenvolvimento, v. 13, n. 73, p. 20-25, 2004.
MEDINA-PAPST, J.; MARQUES, I. Avaliação do desenvolvimento motor de crianças com dificuldades de aprendizagem. Revista Brasileira de Cineantropometria \& Desempenho Humano, v. 12, n. 1, p. 36-42, 2010.

NETO, F. R. et al. Efeitos da intervenção motora em uma criança com Transtorno do Espectro do Autismo. Temas sobre Desenvolvimento, v. 19, n. 105, p. 110-114, 2013.

Manual de avaliação motora. Porto Alegre:

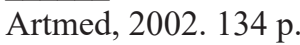

Desenvolvimento Motor de Crianças com Indicadores de Dificuldades na Aprendizagem Escolar. Revista Brasileira Ciência e Movimento, 15, n. 1, p. 45$51,2007$.

O esquema corporal de crianças com dificuldade de aprendizagem. Revista Semestral da Associação Brasileira de Psicologia Escolar e Educacional, v. 15, n. 1, p. 15-22, 2011.

A lateralidade cruzada e o desempenho da leitura e escrita em escolares. Revista CEFAC, 15, n. 4, p. 864-872, 2013.

NETO, S. D. S. et al. A pedagogia do "movimento humano" - O corpo como objeto de estudo. Projeto leitura e escrita: A avaliação motora. p. 22-44, 2005.

PELLEGRINI, A. M. et al. O comportamento motor no processo de escolarização: Buscando soluções para a alfabetização no contexto escolar. Cadernos do Núcleo de Ensino, p. 271-284, 2003.

ROCHA, N. A. C. F.; TUDELLA, E. A influencia da postura sobre o estado comportamental e a coordenacao mao boca do bebe / Behavior of babies in different body posture. Brazilian journal of physical therapy, v. 6, n. 3, p. 167-173, 2002.

RODRIGUES, L. R. Caracterização do desenvolvimento físico, motor e psicossocial de pré-escolares de Florianópolis, SC. 2000. 198f. (Mestrado) - Centro de Educação Física, Fisioterapia e Desportos, Universidade Federal do Estado de Santa Catarina, Florianópolis.

SCHIRMER, C. R.; FONTOURA, D. R.; NUNES, M. L. Distúrbios da aquisição da linguagem e da aprendizagem. Jornal de Pediatria, v. 80, n. 2, p. 95-103, 2004.

URRUTIA, G.; TORTA, S.; BONFILL, X. Metaanálisis (QUOROM). Medicina Clínica, v. 125, supl. 1, p. 32-37, 2005.

Recebido em: 24-07-2018

Aceito em: 12-11-2019 